\title{
Leucocyte esterase test as rapid screen for non-gonococcal urethritis
}

\author{
S A B PERERA (deceased), C JONES, V SRIKANTHA, W RANAWICKRAMA, \\ M N BHATTACHARYYA
}

From the Department of Genitourinary Medicine, Royal Infirmary, Manchester

SUMmaRY The two standard tests for the initial diagnosis of non-gonococcal urethritis (NGU), microscopic examination of gram stained urethral smears and the two glass urine test, have the disadvantage of being insensitive and subjective. The leucocyte esterase test detects enzymes specific to polymorphonuclear leucocytes and can therefore be used as a sensitive indicator of pyuria. This study sought to evaluate its use as a rapid, sensitive, and non-subjective method of screening for NGU.

Of the 81 men with urethral symptoms in the study group, 26 had more than 5 polymorphonuclear leucocytes per high power field $(\times 1000)$ and all 26 were leucocyte esterase test positive; whereas 55 had fewer than 5 polymorphonuclear leucocytes per high power field, but 29 $(53 \%)$ of them had a positive leucocyte esterase test result. In addition, 25 patients in the study group yielded Chlamydia trachomatis on culture. Of these 25, 24 (96\%) were leucocyte esterase test positive, whereas only $11(44 \%)$ were Gram stain positive.

All 40 patients in the control group (without urethral symptoms or signs) were leucocyte esterase test negative.

The leucocyte esterase test is thus a rapid, sensitive, and non-subjective screening aid in the diagnosis of NGU.

The incidence of non-specific genital infection is increasing in patients attending genitourinary medicine clinics in England and Wales. ${ }^{1}$ Non-gonococcal urethritis (NGU) accounts for an appreciable proportion of non-specific genital infection in men. Chlamydia trachomatis is isolated in about $35 \%$ to $55 \%$ of patients with NGU. ${ }^{23}$ The isolation of chlamydiae in cell line culture is still accepted as being the most definitive evidence of the presence of chlamydial infection, although more recently introduced techniques, such as the flourescein labelled monoclonal antibody test for detecting $C$ trachomatis elementary bodies, have the advantage of being more rapid. ${ }^{4}$ In most departments of genitourinary medicine in the UK, however, the initial diagnosis of NGU depends on the interpretation of a Gram stained urethral

\footnotetext{
Address for reprints: Dr M N Bhattacharyya, Department of Genitourinary Medicine, Manchester Royal Infirmary, Oxford Road, Manchester M13 9WL
}

Accepted for publication 13 January 1987 smear and the two glass urine test. These methods have the disadvantage of being subjective, insensitive,${ }^{5}$ and relatively time consuming.

Recent work suggests that pyuria is a sensitive indicator of urethritis. ${ }^{5-7}$ Advances in urine analysis have also provided several new techniques for detecting pyuria, ${ }^{59}$ but most of these are time consuming and expensive. Esterases are leucocyte specific enzymes, ${ }^{10}$ however, and their activity has been found to correlate closely with chamber counts of polymorphonuclear leucocytes. ${ }^{11} \mathrm{~A}$ test that detects urinary esterases should therefore be ideal for detecting pyuria and thus for screening for patients with NGU. A strip test (Boerhinger-Mannheim, Lewes, East Sussex) capable of detecting esterase activity in urine has been introduced. This study aimed at evaluating it as a method of screening for NGU.

\section{Patients and methods}

We studied 81 unselected heterosexual men aged 15 to 70 attending the department of genitourinary medi380 
cine, Manchester Royal Infirmary, in April to August 1986 complaining of urethral symptoms (discharge, dysuria, or both). None had received antibiotics during the previous three months. The control group consisted of $\mathbf{4 0}$ heterosexual men attending the clinic who did not have urethral symptoms or signs.

The patients had not passed urine within two hours before being examined, although the exact time between clinical examination and passing of urine was not recorded in each case. All patients were examined for the presence of urethral discharge before and after gentle massage of the urethra. A swab (Exogen Ltd, Glasgow, Scotland) was inserted 2 or $3 \mathrm{~cm}$ into the anterior urethra, removed, and rolled over an area of $1 \times 2 \mathrm{~cm}$ on a glass slide as well as on to Thayer Martin selective medium. The slide was stained by Gram's method, examined for the presence of Gram negative intracellular diplococci, and then scanned under low power to identify the area of greatest polymorphonuclear leucocyte concentration. This area was then examined with an oil immersion lens, and the mean number of polymorphonuclear leucocytes per high power field $(\times 1000)$ counted for five fields. A polymorphonuclear leucocyte count of 5 or more per high power field was taken as indicating NGU. ${ }^{14}$ Material from the anterior urethra was also examined by wet mount microscopy for Trichomonas vaginalis and yeasts.

Specimens for the culture of $C$ trachomatis were collected by inserting a swab (Exogen) 4 to $5 \mathrm{~cm}$ into the anterior urethra, rotating it, and then placing it into a vial containing phosphate buffer. ${ }^{15}$ Isolation of $C$ trachomatis was carried out in cycloheximide treated Mcoy Cells, and inclusions were identified using an indirect immunofluorescent staining technique after incubation for 60 to 70 hours. ${ }^{16} 17$

Facilities for the culture of Ureaplasma urealyticum were not available for this study.

After the swabs had been taken, segmented urine specimens (first and second voided bladder urine) were obtained using a standard technique, ${ }^{7}$ and were examined for the presence of sinking threads or haziness, or both. In segmented urine specimens collected by this technique, sinking threads or haziness in the first specimen and clear urine in the second specimen were taken to be indicative of urethritis.

The leucocyte esterase test was then performed on both urine specimens. A plastic strip fitted at one end with a filter paper pad containing indoxyl carboxylic acid ester was dipped briefly (no longer than one second) into the urine specimen. The test zone was compared with a colour scale at 60 seconds, and equivocal results were checked at 120 seconds. Leucocyte esterase catalyses the hydrolysis of indoxyl carboxylic acid ester to indoxyl, which then reacts with a diazonium salt to produce a purple colour, ${ }^{11} 18$ indicating a pos- itive result. This result is independent of cell lysis.

Patients were considered to have NGU if leucocyte esterase test results were positive for first and negative for second voided bladder urine specimens.

In patients for whom a diagnosis had been made, standard treatment regimens were instituted, contact tracing performed, and tests for cure carried out after completion of treatment.

Standard serological tests for syphilis were performed on all patients.

\section{Results}

The study and control groups were demographically similar. All routine tests, other than those directly related to NGU, gave negative results in the study group. Two patients in the control group had positive C trachomatis cultures, but other screening tests were negative.

Table 1 shows the results of the various tests. Only 26 of the 81 patients in the study group had more than 5 polymorphonuclear leucocytes per high power field ( $\times 1000)$ in their urethral smears stained by Gram's method. All 26 of these patients had positive leucocyte esterase test results.

Table 1 Results of diagnostic tests in 81 patients with urethral symptoms

\begin{tabular}{lll}
\hline Diagnostic test & No (\%) positive & No (\%) negative \\
\hline Gram stained smear & $26(32)$ & $55(68)$ \\
Two glass urine test & $32(40)$ & $49(60)$ \\
Leucocyte esterase test & $55(68)$ & $26(32)$ \\
$\begin{array}{l}\text { Chlamydia trachomatis } \\
\text { culture }\end{array}$ & $25(31)$ & $56(69)$ \\
\hline
\end{tabular}

Of the 55 patients in the study group whose urethral smears had fewer than 5 polymorphonuclear leucocytes per high power field, and who would therefore not be diagnosed as having NGU by usually accepted criteria, 29 (53\%) had positive leucocyte esterase test results. All 29 had urethral symptoms, and 13 had definitive evidence of a positive urethral culture of $C$ trachomatis.

On clinical examination 36 patients had a urethral discharge and 15 of them yielded $C$ trachomatis cultures. All 36 had positive leucocyte esterase test results. Of the 45 without a clinically obvious discharge, 19 were leucocyte esterase test positive, and eight of these patients yielded $C$ trachomatis.

On the basis of the two glass urine test, $32(40 \%)$ of the study group had urethritis, and all had positive leucocyte esterase test results. Of the 49 patients with a negative two glass urine test result, 22 had positive leucocyte esterase test results, and 13 of these 22 
yielded $C$ trachomatis.

Table 2 shows that of the total of 25 patients yielding $C$ trachomatis, 24 had positive leucocyte esterase test results, whereas only 11 had positive Gram stained urethral smears.

Although none of the 40 patients in the control group had a positive leucocyte esterase test result in either first or second voided bladder urine specimens, two yielded $C$ trachomatis on culture.

None of the patients with urethral symptoms had positive leucocyte esterase test results for first voided bladder urine specimens.

Table 2 Relation between 25 positive cultures of Chlamydia trachomatis and the other diagnostic test results

\begin{tabular}{lll}
\hline Diagnostic test & No (\%) positive & No (\%) negative \\
\hline Culture for C trachomatis & $25(100)$ & 0 \\
Leucocyte esterase test & $24(96)$ & $1(4)$ \\
Gram stained smear & $11(44)$ & $14(56)$ \\
\hline
\end{tabular}

\section{Discussion}

Since its introduction, the leucocyte esterase test strip has been used to diagnose upper urinary tract infections, ${ }^{131920}$ and to screen peritoneal fluid in the early diagnosis of peritonitis. ${ }^{21}$ Leucocytes contain several esterases that are not present in serum, urine, or kidney tissue. ${ }^{10}$ Leucocyte esterase activity is not influenced by urinary $\mathrm{pH}$, proteins, underlying renal disease, altered renal function, or commonly used drugs, ${ }^{11}$ and has been found to relate closely to chamber counts of polymorphonuclear leucocytes. ${ }^{11}$ These features would appear to make the leucocyte esterase test ideal to detect pyuria, and consequently to screen patients for NGU.

In this study we found that the leucocyte esterase test identified all 26 patients with a positive urethral smear, and also provided a positive diagnosis of urethritis in $29(53 \%)$ of 55 patients with negative smears. Of these 29 men, 13 had positive $C$ trachomatis cultures. These results are similar to those of Desai and Robson, who also noted that $45 \%$ of patients diagnosed as having NGU on microscopic examination of urine had fewer than 4 polymorphonuclear leucocytes per high power field $(x$ 1000 ) in Gram stained urethral smears. ${ }^{8}$

In the present study, $36(65 \%)$ patients had urethral discharge and each had a positive leucocyte esterase test result. This supports Swartz's conclusion that any urethral discharge is objective evidence of NGU. Not all men with NGU have a urethral discharge, however. $^{23}$ In addition to these 36 patients, a further 18 without urethral discharge were identified as having
NGU on the basis of the leucocyte esterase test. None of these had more than 5 polymorphonuclear leucocytes per high power field $(\times 1000)$ in Gram stained urethral smears, but nine had positive $C$ trachomatis cultures.

Naked eye examination of 81 first voided urine specimens showed haziness or sinking threads, or both, in 32 patients (table 1). All had positive leucocyte esterase test results. Naked eye examination, however, failed to indicate pyuria in 22 more patients who had positive leucocyte esterase test results. $C$ trachomatis was isolated from the urethras of 13 of these 22 patients. Again the leucocyte esterase test appeared to be more accurate than the two glass urine test in detecting urethritis.

Of the 25 patients who had $C$ trachomatis positive cultures, 24 had positive leucocyte esterase test results, whereas only 11 had positive Gram stained urethral smears (table 2). The accuracy of the leucocyte esterase test $(24 / 25,96 \%)$ thus appears to be appreciably greater than that of urethral smears stained by Gram's method $(11 / 25,44 \%)$ in screening for $C$ trachomatis positive urethritis.

Of the 40 patients in the control group who were free of urethral signs or symptoms, two had positive $C$ trachomatis cultures. The leucocyte esterase test failed to give positive results in these two cases. This seems to be at variance with other studies in which asymptomatic patients with positive $C$ trachomatis cultures showed the presence of pyuria. ${ }^{5924}$ The absence of signs and symptoms probably reflected a low level of urethral inflammation and, as the leucocyte esterase test depends on the presence of at least a minimal inflammatory response, may explain these false negative results. This does, however, indicate a limitation of the leucocyte esterase test.

Early consultation and a relatively short interval between examination and last passing urine probably accounts for the low proportion of patients with positive Gram stained urethral smears and demonstrable urethral discharge seen in this study. These two factors are less important in the leucocyte esterase test, as it detects even minimal pyuria. It is thus a more sensitive screening test in such circumstances, and its use may obviate the need for repeat visits to give early morning specimens. Simmons showed the importance of treating patients at their first visit, as $10 \%$ of his patients failed to return for their follow up appointments. ${ }^{25}$

The leucocyte esterase test cannot, however, replace the Gram stained urethral smear, as the latter is necessary to exclude Gram negative intracellular diplococci. This study showed that the leucocyte esterase test, in conjunction with the urethral smear stained by Gram's method, provides a simple screening test for NGU. The leucocyte esterase test, with its 
$96 \%$ sensitivity, identifies on their first visit almost all patients who need treatment for NGU, thereby preventing delay in treatment and contact tracing and any unwanted sequlae. ${ }^{26}$

We thank Drs KR Haye, TK Chatterjee, and FH Chowdhury, consultants in genitourinary medicine, for allowing access to their patients and Mr R Speakman, charge nurse, for his help in collecting urine specimens. We also thank Boehringer-Mannheim for providing the leucocyte esterase test strips.

\section{References}

1 Communicable Disease Surveillance Centre. Sexually transmitted diseases surveillance in Britain: 1984. Br Med J 1986;293:942-3.

2 Oriel JD, Ridgway GL. Genital infections by Chlamydia trachomatis. London: Edward Arnold, 1982:41-8.

3 Taylor-Robinson D, Thomas BJ. The role of Chlamydia trachomatis in genital tract and associated diseases. J Clin Pathol 1980;33:205-33.

4 Thomas BJ, Evans RT, Hawkins DA, Taylor-Robinson D. Sensitivity of detecting Chlamydia trachomatis elementary bodies in smears by use of flurescein labelled monoclonal antibody: comparison with conventional chlamydial isolation. J Clin Pathol 1984;37:812-6.

5 Oriel JD, Reeve P, Wright JT, Owen J. Chlamydial infection of male urethra. British Journal of Venereal Diseases 1976; 52:46-51.

6 Richmond SJ, Hilton AL, Clark SKR. Chlamydial infection: role of Chlamydia Subgroup A in non-gonococcal and postgonococcal urethritis. British Journal of Venereal Diseases 1972;48:437-44.

7 Perera SAB. Use of Kora-Slide II with grid and uncentrifuged segmented urine specimens in the diagnosis of non-gonococcal urethritis: a quantitative technique. Sex Transm Dis 1985; 12:14-8.

8 Desai K, Robson HG. Comparison of Gram-stained urethral smears and first-voided urine sediment in the diagnosis of nongonococcal urethritis. Sex Transm Dis 1982;9:21-5.

9 Adger H, Shafer MA, Sweet RL, Schachter J. Screening for Chlamydia trachomatis and Neisseria gonorrhoeae in adolescent males: value of first catch urine examination. Lancet 1984;ii:944-5.

10 Rindler-Ludwig R, Schmalze F, Braunstner H. Esterases in human neutrophil granulocytes: evidence for their protease nature. Br J Haematol 1974;27:57-64.
11 Kusumi RK, Grover PG, Kunin CM. Rapid detection of pyuria by leucocyte esterase activity. JAMA 1981;245:1653-5.

12 Hale DC, Wright DI, McKie JE, Isenberg HD, Jenkins RD, Matsen JM. Rapid screening for baceriuria by light scatter photometry (Autobac): a collaborative study. J Clin Microbiol 1981:13:147-50.

13 Wallis C, Melnick JL, Longoria CJ. Colorimetric method for rapid determination of bacteriuria. J Clin Microbiol 1981; 14:342-6.

14 Schachter J. Chlamydia trachomatis infections: epidemiology and disease spectrum. In: Oriel JD, Harris JRW, eds. Recent advances in sexually transmitted diseases. 3rd ed. London: Churchill Livingstone, 1986:39-44.

15 Darougar S, Jones BR, Kinnision JR, Vaughan-Jackson JD, Dunlop EMC. Chlamydial infection: advances in the diagnostic isolation of Chlamydia, including TRIC agent, from the eye, genital tract, and rectum. British Journal of Venereal Diseases 1972;48:416-20.

16 Gordon FB, Harper IA, Quan AL, Trehan JD, Dwyer RSC, Garland JA. Detection of Chlamydia (Bedsonia) in certain infections of man. Laboratory procedures: comparison of yolk sac and cell culture for detection and isolation. $J$ Infect Dis 1969;126:451-62.

17 Ghadirian FD, Robson HG. Chlamydia trachomatis genital infections. British Journal of Venereal Diseases 1979;55:415-8.

18 Abbasi IA, Hess LW, Johnson TR, McFadden E, Chernow B. Leucocyte esterase activity in rapid detection of urinary tract and lower genital tract infections in obstetric patients. Am J Perinatol 1985;2:311-3.

19 Perry JL, Mathers JS, Weesner DE. Evaluation of leucocyte esterase activity as a rapid screening technique for bacteriuria. J Clin Microbiol 1982;15:852-4.

20 Smalley DL, Dittman AN. Use of leucocyte esterase-nitrite activity as predictive assays of significant bacteriuria. J Clin Microbiol 1983;18:1256-7.

21 Chan LK, Oliver DO. Simple method for early detection of peritonitis in patients on continuous ambulatory peritoneal dialysis. Lancet 1979;ii:1336-7.

22 Swartz SL, Kraus SJ, Herman KL, Stragel MD, Brown WJ, Allen SD. Diagnosis and etiology of non-gonococcal urethritis. $J$ In fect Dis 1978;136:445-54.

23 Jacobs NF, Kraus SJ. Gonococcal and non-gonococcal urethritis in men. Clinical and laboratory differentiation. Ann Intern Med 1975;82:7-12.

24 Holmes KK, Handsfield HH, Wang SP, et al. Etiology of nongonococcal urethritis. $N$ Engl J Med 1975;292:1199-205.

25 Simmons PD. Evaluation of early morning smear investigation. British Journal of Venereal Diseases 1978;54:128-9.

26 Hawkins DA, Taylor-Robinson D, Thomas BJ, Harris JRW. Microbiological survey of acute epididymitis. Genitourin Med 1986;62:342-4. 